\title{
Modeling the impact of a biocontrol agent, Puccinia lagenophorae, on interactions between a crop, Daucus carota, and a weed, Senecio vulgaris
}

\author{
J. Frantzen ${ }^{\mathrm{a}, *}$, H. Müller-Schärer ${ }^{\mathrm{b}}$ \\ ${ }^{a}$ Experimental Plant Ecology, Department of Ecology, University of Nijmegen, P.O. Box 9010, 6500 GL Nijmegen, The Netherlands \\ ${ }^{\mathrm{b}}$ Unit of Ecology and Evolution, Department of Biology, University of Fribourg, CH-1700 Fribourg, Switzerland
}

\begin{abstract}
The impact of a biocontrol agent spreading from a point source on crop-weed interactions was modeled. The model encompassed: (i) severity of crop-weed competition as affected by a rust pathogen, (ii) velocity of spread of the rust pathogen, and (iii) density of infected plants introduced in the weed population as starting points (inoculum sources) for an epidemic. The model was parameterized for a study system encompassing the crop Daucus carota (carrot), the weed Senecio vulgaris (common groundsel), and its antagonist Puccinia lagenophorae. The parameters of (i) were estimated in a greenhouse study using a response-surface design. Estimates of the parameter of (ii) were obtained from the literature. The density of infected plants (iii) was varied to simulate crop loss as function of density. Simulations were run for various densities of the weed and various velocities of rust pathogen spread. The results of the simulations indicated a croploss ranging from 5 to $10 \%$ at levels of relatively weak $D$. carota-S. vulgaris competition. Density of inoculum sources and velocity of $P$. lagenophorae spread had only minor effects on crop loss. In contrast, density of inoculum sources and velocity of spread had major effects on crop loss at levels of intermediate (range of 10-35\% loss) and severe competition (range of 30-70\% loss). The results are discussed both with respect to biological control of $S$. vulgaris using $P$. lagenophorae as biocontrol agent and as a general model describing the impact of the spatial dynamics of a pathogen (natural enemy) on plant competition.
\end{abstract}

Keywords: Plant competition; Spatial dynamics; Rust pathogen; Biological weed control; System-management approach; Senecio vulgaris; Puccinia lagenophorae

\section{Introduction}

The system-management approach to biological control of weeds evolved from a theoretical concept (Müller-Schärer and Frantzen, 1996) to research close to practical application (Grace and Müller-Schärer, 2003). This approach is based on the introduction of some infected weed plants as an inoculum source into a weed population, and the subsequent spread of the antagonist over the entire weed population (Frantzen and van den Bosch, 2000). Such an

\footnotetext{
* Corresponding author. Fax: +31 206425085 .

E-mail addresses: j.frantzen@uicbme.com, j.frantzen@vumc.nl (J. Frantzen).
}

epidemic results in reduced competition between crop and weed (Frantzen, 2000). Whether this relief is sufficient to prevent crop losses depends on (i) the severity of cropweed competition as affected by a pathogen, (ii) the velocity of spread of the rust pathogen, and (iii) the density of infected plants introduced (Frantzen et al., 2001). An outline for a crop-loss model including these factors was proposed. Here, we shall establish and parameterize it for a system composed of the crop Daucus carota L., the weed Senecio vulgaris L., and the latter's natural antagonist Puccinia lagenophorae Cooke.

Severity of plant competition can be quantified using various methods. Here, we use a so-called response-surface analysis (Firbank and Watkinson, 1990) as extended by 
Frantzen et al. (2001) to measure the impact of $P$. lagenophorae on $D$. carota-S. vulgaris competition. No other study has been reported yet that quantifies the impact of a pathogen on plant competition using response-surface analysis. Application of this design is labor intensive, but unless other methods, it provides insight into the impact of a pathogen on plant competition over a large range of densities and proportions of the competing plant species.

The velocity of spread of $P$. lagenophorae is about 8-10 $\mathrm{cm}$ per day assuming a radial expansion from a point source at a constant velocity (Frantzen et al., 2001, 2002). The velocity can also increase linearly, or even exponentially, with time resulting in velocities of spread $>10 \mathrm{~cm}$ (Frantzen and van den Bosch, 2000). In the model presented here, we shall include slower and faster spread of $P$. lagenophorae to account for variability in velocity of spread in the field.

Severity of plant competition and velocity of spread of the rust pathogen set the scene for biological control following the system-management approach. Both severity and velocity will depend on prevailing biotic and abiotic conditions at a farm. Neither biotic factors like the genotype of the weed nor abiotic conditions like temperature are easy to manage by a farmer. The easiest way to speed up infection of all plants within a population is to introduce additionally infected plants as inoculum sources. The density of inoculum sources is, therefore, an essential factor to achieve successful biological control. We shall simulate loss of $D$. carota as function of density of inoculum sources given various levels of severity of $D$. carota-S. vulgaris competition and various velocities of $P$. lagenophorae spread.

\section{Materials and methods}

\subsection{Study system}

The crop D. carota is sown from early spring until autumn and depending on variety, carrots can be harvested within 2-6 months after sowing. Here, we used the variety Napoli that can be harvested within 2-3 months after sowing under common field conditions in Switzerland.

The predominantly selfing annual $S$. vulgaris is an especially serious weed in crops with relatively short growing periods like $D$. carota, i.e., under conditions with a high disturbance frequency causing bare soil. Crop losses due to abundance of $S$. vulgaris are commonly reported by farmers, but have so far only been reported in the literature for Lactuca sativa L., lettuce, (Paul and Ayres, 1987) and D. carota, carrots (this study). The Swiss maternal line ELS of $S$. vulgaris (Wyss, 1997) was used throughout the study.

The life cycle of the rust pathogen $P$. lagenophorae is comprised of the asexual stage of aecidia within Europe (Frantzen and Müller-Schärer, 1999). The fungus survives as mycelium within plants of $S$. vulgaris over winter, aecidiospores are produced within the aecidia in spring, and these aecidiospores spread to other parts of the infected plants or other plants within a population. The mortality rate of $P$. lagenophorae over winter is very high and, in general, no or only a few infected S. vulgaris plants are present to serve as inoculum sources for a $P$. lagenophorae epidemic within a $S$. vulgaris population in spring (Frantzen and Müller-Schärer, 1999; Leiss and Müller-Schärer, 2001; Paul and Ayres, 1986). Increasing the number of infected plants, i.e., the number of inoculum sources, within a $S$. vulgaris population in spring is one way to induce and stimulate $P$. lagenophorae epidemics controlling $S$. vulgaris. The Swiss line ELS of $P$. lagenophorae, derived from a single aecidiospore (Wyss, 1997), was used throughout the study.

\subsection{Model}

The average yield per crop plant, $w_{c r}$, at any harvest time $t$ can be described as a function of intraspecific competition, interspecific competition with a weed we, and impact of a pathogen on competitiveness of the weed (Frantzen et al., 2001),

$w_{c r, t}=w_{m, c r, t} / 1+\alpha_{t}\left(N_{c r}+\varepsilon_{t} r_{t} N_{w e}\right)$,

where $w_{m}$ is the estimated maximum yield (in g) of a crop plant $\mathrm{cr}$ at harvest time $t$ (in days after sowing) growing without competitors, $\alpha$ (in $\mathrm{cm}^{2}$ ) is a competition coefficient describing intraspecific competition of the crop, $N$ is plant density (in number per $\mathrm{cm}^{2}$ ) of crop and weed, respectively, $\varepsilon$ the ratio of intra- and interspecific competition, and $r$ the reduction ratio of interspecific competition due to attack of the weed by a natural enemy. The possible range of $r$ is $0-1$. Intuitively, the value of $r$ will be lower the longer a plant is attacked by a pathogen. For example, if parameter $r$ is a linear function of period of attack as suggested by the results presented here (see Section3), equation (1) becomes,

$w_{c r, t}=w_{m, c r, t} / 1+\alpha_{t}\left(N_{c r}+\varepsilon_{t}\left(1-b\left(t-t^{\prime}\right)\right) N_{w e}\right)$,

where $t^{\prime}$ is the time of attack (in days after sowing) of the weed by a natural enemy and, assuming the attack continues as is the case for pathogens like $P$. lagenophorae, $t-t^{\prime}$ is the period of attack by a natural enemy until harvest of the crop. The regression parameter $b$ indicates the decrease of $\varepsilon_{t}$, the longer the period of attack by the rust pathogen is. In biological terms, the damage to the weed will be larger the longer it is attacked by the rust pathogen.

The area of a host population occupied by a fungus spreading at a constant velocity from an inoculum source is after a time $t^{\prime}$ (van den Bosch et al., 1988)

$A=\pi\left(c t^{\prime}\right)^{2}$,

where $c$ is the velocity of spread in $\mathrm{cm}$ per day. In turn, the time can be calculated at which a specific area $A\left(\mathrm{~cm}^{2}\right)$ of the host population is infected. Assuming a uniform distribution of inoculum sources, the area $A$ is proportional to the density of inoculum sources and equation (2) becomes,

$$
w_{c r, t}=w_{m, c r, t} / 1+\alpha_{t}\left(N_{c r}+\varepsilon_{t}\left(1-b\left(t-\frac{\sqrt{\frac{\pi}{N_{i}}}}{c}\right)\right) N_{w e}\right) \text {, }
$$


where $N_{i}$ is the density of inoculum sources in number per $\mathrm{cm}^{2}$.

The average yield per crop plant without interspecific competition would be,

$w_{c r, t}=w_{m, c r, t} /\left(1+\alpha_{t} N_{c r}\right)$.

Crop loss $Y$ at harvest time $t$ (in days after sowing) can then be defined as,

$Y_{t}=\left[1-\frac{\text { equation }(4)}{\text { equation }(5)}\right] \times 100 \%$

\subsection{Competition parameters}

Competition parameters $\alpha, \varepsilon$, and $r$ were estimated for the study system in a greenhouse experiment. Seeds of $S$. vulgaris and D. carota were sown into 11-cm-diameter pots filled with nutrient-amended peat (Floragard, TKS 2). A range of densities and proportions of seeds of the two species were assigned to the pots based on a series of 5, 15, 25, $35,45,55$, and 65 seeds of each species resulting in $7^{2}=49$ combinations, and each combination was randomly assigned to a pot. In the following, we refer to the 49 pots as one series. Four series were established and the pots of these four series were randomly assigned to a position on a bench in the greenhouse on March 7, 2000. Pots were kept wet by watering from below. In addition, seeds were sprayed daily with distilled water in the first week after sowing to stimulate seed germination and establishment of plants. Series of plants were inoculated with aecidiospores of $P$. lagenophorae, (i) at 2 and 3 weeks after sowing, (ii) only at 3 weeks after sowing, (iii) and (iv) not inoculated serving as controls. Aecidiospores were sprayed with a de Vilbiss sprayer and plastic bags subsequently covered pots for about $16 \mathrm{~h}$ to maintain high humidity for germination of the spores and subsequent penetration of a host plant by the fungus. Plastic bags also covered plants of the noninoculated series during this period. The light period in the greenhouse was fixed at $16 \mathrm{~h}$ a day by using supplemental light of HPI-bulbs in the early morning and early evening. Light intensity, temperature, and relative air humidity were recorded continuously. One control series was harvested at 4 weeks after sowing, but maximum biomass per pot was not yet achieved. This series was, therefore, discarded from further analysis. The other three series were harvested at 7 weeks after sowing and plants of $S$. vulgaris produced abundant seed at that time. The numbers of $D$. carota and $S$. vulgaris plants were determined for each pot and the fresh weight of the D. carota roots, i.e., the carrots.

Competition parameters $\alpha, \varepsilon$, and $r$ were estimated using equation (1) in an iterative procedure of nonlinear regression. The routine of the Statistical Package for Social Sciences (SPSS, version 10) was used. Estimates of the parameter $r$ were linearly regressed against the period of infection $t-t^{\prime}$ to obtain parameter $b$ (cf. equation 1 and 2). Parameter $r$ was set at 1 for plants not infected, i.e., $t-t^{\prime}$ equals zero. A linear model was chosen being the simplest model having only three points to fit a model.

\subsection{Simulations}

Crop loss as function of the inoculum density $D$ was simulated using equation (6) and the best estimates of the parameters $\alpha, \varepsilon$, and $b$ from the greenhouse experiment (see above). Simulations were run using a density of 350 D. carota plants per $\mathrm{m}^{2}$, which is a commercially used density of this crop. Density of $S$. vulgaris was 100,350 , or 1000 plants per $\mathrm{m}^{2}$ representing relatively weak, intermediate, and severe crop-weed competition. The velocity of spread of $P$. lagenophorae was set at $5 \mathrm{~cm}$ per day, $10 \mathrm{~cm}$, or $20 \mathrm{~cm}$ representing a relatively slow, intermediate, and fast spread. The velocity of $10 \mathrm{~cm}$ per day is the best estimate of a study presented elsewhere (Frantzen et al., 2001, 2002).

\section{Results}

\subsection{Competition parameters}

On average, $62 \%$ of $D$. carota seeds germinated per pot in the greenhouse study. The range of germination was 9$100 \%$. Sixty-eight percent of S. vulgaris seed germinated on average per pot with a range of $0-100 \%$. Correlations between number of seeds sown per pot and number of plants present at harvest were relatively high, i.e., Pearson's correlation coefficient within a range of $0.82-0.93$ depending on species and series, and all correlations were significant $(P<0.001)$. Seed densities were entered into Eq. (1) and predictions of carrot weight based on this model well fitted data of observed carrot weight as indicated by an approximate linear relationship between predicted and observed carrot weight (Fig. 1). Interspecific competition was more severe than intraspecific competition as indicated by a value of $\varepsilon$ significantly higher than 1 (Table 1 ). Infection of $S$. vulgaris by $P$. lagenophorae reduced severity of interspecific competition as indicated by values of parameter $r$ significantly lower than 1 . The impact of $P$. lagenophorae on interspecific competition was larger when $S$. vulgaris plants became infected relatively early, i.e., $r_{t 1}$ lower than $r_{\mathrm{t} 2}$, but this effect was not significant.

Regressing the parameter $r$ against the period of infection resulted in a regression slope $b$ of 0.02 (SE 0.001) and the slope differed significantly $(P<0.01)$ from zero. The regression was based on only three points, the parameters $r_{\mathrm{t} 1}, r_{\mathrm{t} 2}$, and $r=1$ if plants were not infected.

\subsection{Simulations}

Crop loss could be reduced to a level below $20 \%$ using a density of 2 to 3 inoculum sources per $10 \mathrm{~m}^{2}$ at relatively weak crop-weed competition (Fig. 2A). The density of inoculum sources and the velocity of $P$. lagenophorae spread had a minor effect on crop loss. In contrast, density 


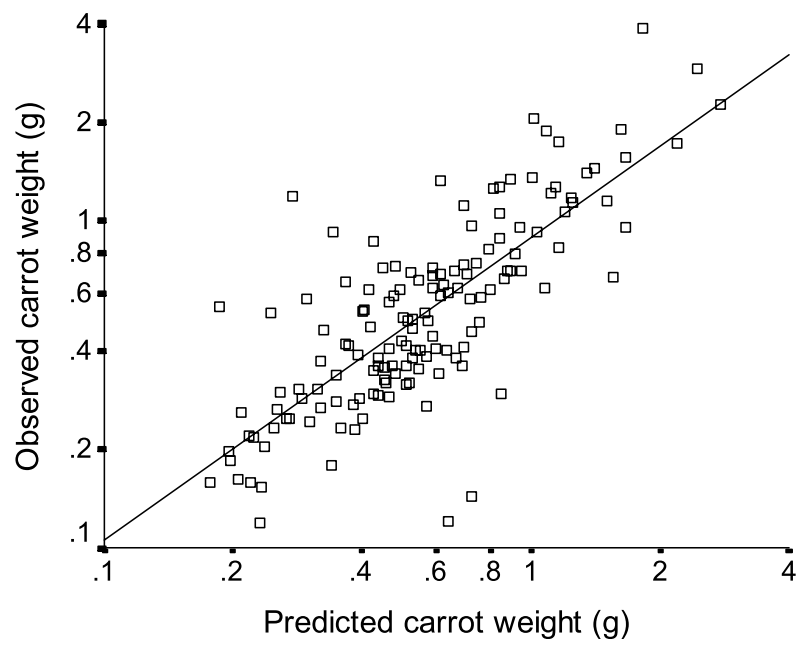

Fig. 1. Observed and predicted weight of carrots in a greenhouse study of the competitive interaction between the crop D. carota and the weed $S$. vulgaris as affected by the pathogen P. lagenophorae. Predictions based on a model of which the parameters are presented in Table 1. Data presented on a log-log scale to better distinguish individual points.

Table 1

Estimates and $95 \%$ confidence bounds of parameters of a competition model $^{a}$ for Daucus carota in competition with Senecio vulgaris

Parameter $\quad$ Estimate $\quad 95 \%$ Confidence interval

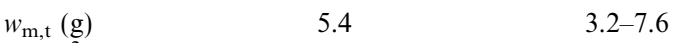

$\alpha\left(\mathrm{cm}^{2}\right) \quad 13.2 \quad 6.1-20.4$

$\varepsilon \quad 2.2$

$1.2-3.3$

$0.16 \quad 0.09-0.24$

\begin{tabular}{lll}
$r_{\mathrm{t} 1}$ & 0.16 & $0.09-0.24$ \\
$r_{\mathrm{t} 2}$ & 0.33 & $0.18-0.49$ \\
\hline
\end{tabular}

${ }^{\mathrm{a}} w_{c r, t}=w_{m, c r, t} /\left(1+\left(\alpha_{t} \times\left(N_{c r}+\left(\varepsilon_{t} \times r_{t} \times N_{w e}\right)\right)\right)\right)$.

Where $w_{\text {cr, t }}$ is the average weight of a carrot in a pot at $t=7$ weeks after sowing, $w_{\mathrm{m}}$ is the estimated maximum weight of a carrot from a crop plant growing without competitors, $\alpha$ a competition coefficient describing intraspecific competition, $N$ seed density of $D$. carota and $S$. vulgaris, respectively, $\varepsilon$ the equivalence of interspecific competition, and $r$ the reduction of interspecific competition due to attack of the weed by a natural enemy.

${ }^{\mathrm{b}}$ Plants of $S$. vulgaris were relatively early inoculated and inoculation was repeated $\left(r_{\mathrm{t} 1}\right)$, or plants were inoculated relatively late and only once $\left(r_{\mathrm{t} 2}\right)$.

of inoculum sources and velocity of spread had major effects at levels of intermediate competition (Fig. 2B). Two inoculum sources per $10 \mathrm{~m}^{2}$ were sufficient to keep crop loss within $20 \%$ at a relatively fast spread of $20 \mathrm{~cm}$ per day whereas 10 were required at a velocity of $10 \mathrm{~cm}$ per day. A slow spread of $P$. lagenophorae did require more than 10 sources 10 per $\mathrm{m}^{2}$ to keep crop loss at $20 \%$. At levels of strong competition, more than 10 inoculum sources per $10 \mathrm{~m}^{2}$ were required to keep loss at $20 \%$ independent of velocity of $P$. lagenophorae spread (Fig. 2C).

\section{Discussion}

Five parameters were included in the biocontrol model presented here, $w_{\mathrm{m}}, \alpha, \varepsilon, b$, and $c$. Biotic and abiotic factors
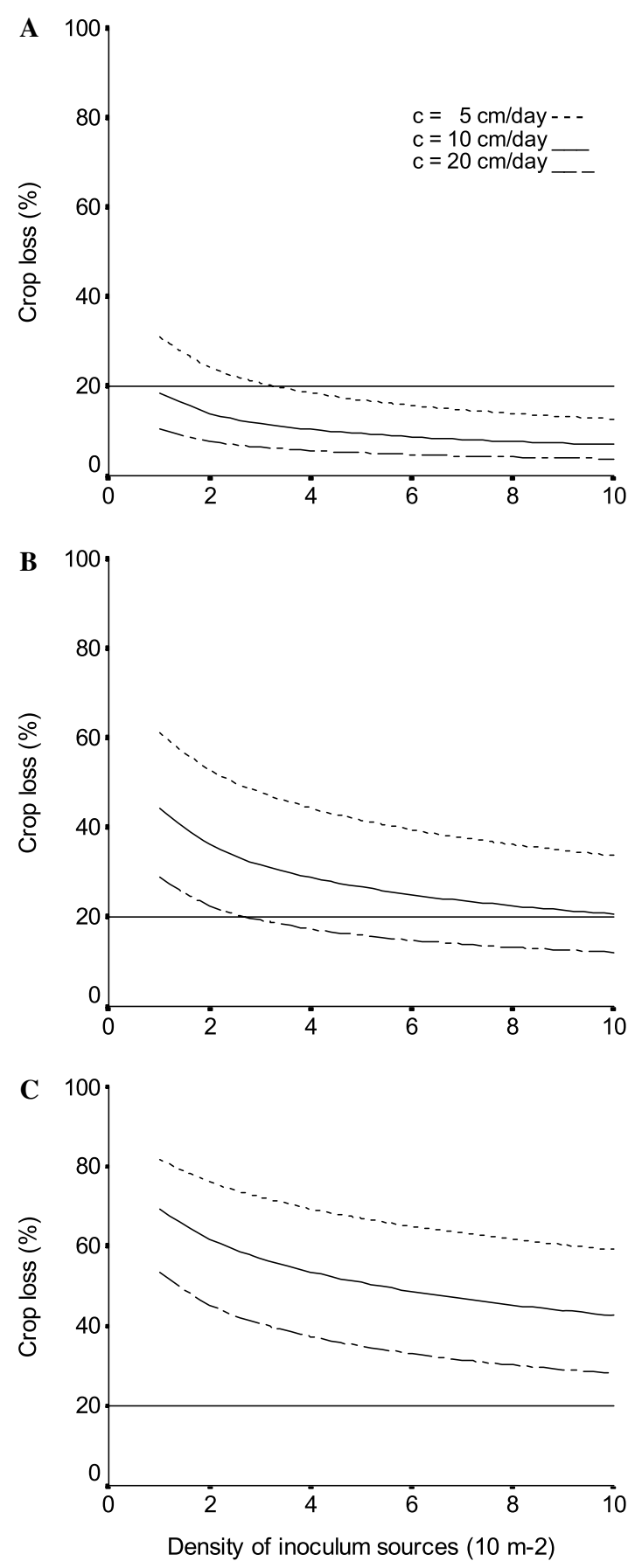

Fig. 2. Simulations of crop loss as function of density of $P$. lagenophorae inoculum sources. Simulations at relatively mild levels of crop-weed competition, i.e., $100 \mathrm{~S}$. vulgaris plants per $\mathrm{m}^{2}$ (A), intermediate competition, i.e., $350 \mathrm{~S}$. vulgaris plants per $\mathrm{m}^{2}(\mathrm{~B})$, and severe competition, i.e., $1000 \mathrm{~S}$. vulgaris plants per $\mathrm{m}^{2}(\mathrm{C})$, and various velocities of $P$. lagenophorae spread. The straight line indicates $20 \%$ crop loss and it has been added to make the differences between (A), (B), and (C) clearer.

influence each of the parameters. All parameters were estimated under one set of conditions either in the competition experiment presented here or a study presented elsewhere (Frantzen et al., 2001, 2002). The estimates of the parameters may, therefore, differ among sets of conditions. However, crop loss of $D$. carota as a function of inoculum 
source density was simulated varying both the severity of crop-weed interaction, as expressed by the density of the weed $S$. vulgaris, and the velocity of spread of the biocontrol agent $P$. lagenophorae, as expressed in $c$. The variation in these two key components of the model may already reflect much environmental variation influencing the five model parameters mentioned above. We therefore assume that some general statements about the efficacy of $P$. lagenophorae as biocontrol agent of $S$. vulgaris in $D$. carota crops can be made.

The biotrophic pathogen $P$. lagenophorae requires living host tissue to grow and reproduce. It can, therefore, not be produced in large amounts limiting the number of inoculum sources that can be used to control a $S$. vulgaris population. The system-management approach to biological control was especially proposed for the use of such biotrophic organisms as biocontrol agents (MüllerSchärer and Frantzen, 1996). Here, we assumed that a density of $P$. lagenophorae inoculum sources higher than 10 per $10 \mathrm{~m}^{2}$ is out of practical order. Keeping this limit in mind, $P$. lagenophorae epidemics can be used for $S$. vulgaris control at levels of weak competition between S. vulgaris and D. carota accepting $5-10 \%$ crop loss. A relatively low severity of competition can be expected at low $S$. vulgaris density, i.e., 100 plants per $\mathrm{m}^{2}$, as simulated in the present study. At an intermediate level of competition, biocontrol is only possible accepting higher levels of crop loss, i.e., $10-35 \%$. Only at a relatively fast spread of $P$. lagenophorae, i.e., $20 \mathrm{~cm}$ per day, crop loss may be around $10 \%$. Such a velocity is not unrealistic (cf. Frantzen and van den Bosch, 2000), but we do not know yet the range of conditions under which such a velocity can be achieved. At a level of severe competition crop loss will be at least 30\%. Such levels of crop loss were indeed observed in field experiments conducted at five farms in Switzerland (Grace and Müller-Schärer, 2003).

The use of $P$. lagenophorae for control of $S$. vulgaris was suggested nearly 20 years ago by Paul and Ayres (1986). In a series of studies they demonstrated the negative impact of $P$. lagenophorae on $S$. vulgaris. Among others, a strong reduction of interference was demonstrated between $S$. vulgaris and, the wild plant Euphorbia peplus L. (Paul, 1989), the crop L. sativa L. (Paul and Ayres, 1987), and the weed Capsella bursa-pastoris (L.) Medic. (Paul and Ayres, 1990). The negative impact was always determined for $S$. vulgaris plants infected completely and at a relatively early stage of development. The simulations of the present study indicate that this early and complete infection is a conditio sine qua non for achieving a zero level of crop loss. However, the use of $P$. lagenophorae as biocontrol agent may be complementary in situations where chemical control does not, or insufficiently, control S. vulgaris (Frantzen et al., 2002). In such situations, even a crop loss of $30 \%$ might be acceptable knowing the low costs of using $P$. lagenophorae (Frantzen et al., 2001) and the high level of crop loss having no control of $S$. vulgaris at all.
The model presented here might serve as a general model describing the impact of the spatial dynamics of a natural enemy on plant competition. The empirical part of the model describing crop-weed interactions was proposed by Watkinson (1981) and subsequently extended by Frantzen et al. (2001) to include effects of a natural enemy on interspecific competition. The model without this extension has been thoroughly validated and it is suitable to describe competition between species over a large range of plant densities and proportions taking into account intra- and interspecific competition (Law and Watkinson, 1987; Watkinson and Freckleton, 1997). Here, we present data to validate the extended model with respect to the impact of a natural enemy on the competitive interactions. The model fitted well and parameters of the model could be estimated quite precisely. Estimates of the parameter $r$ expressing the impact of a natural enemy on interspecific competition could be obtained for the first time and these indicated a significant impact of $P$. lagenophorae on competition between $D$. carota and $S$. vulgaris. The parameter $r$ was closely related to the period that plants were infected, i.e., the longer the period of infection the smaller the value of $r$ and thus the more reduction of interspecific competition as expressed by parameter $\varepsilon$. The estimated linear relationship should be regarded with some caution. Only a few data points were available and a linear relationship close to $r=0$ is unlikely as $P$. lagenophorae could not kill the host under the conditions of the greenhouse experiment. The relationship between parameter $r$ and the period of infection, or period of attack by an insect, needs to be investigated with respect to other systems. Once a function describing the dynamics of the impact of a natural enemy on plant competition established, the spatial dynamics of the natural enemy, as expressed in the velocity of spread $c$, can be included in the model. Including parameter $c$ assumes spread of an organism at a constant velocity. This assumption holds for many organisms after an initial period of spread at accelerating velocity (Shigesada and Kawasaki, 1997; but see Frantzen and van den Bosch, 2000).

As for any model, the model presented here does not serve as an exact description of reality. The interactions between the three organisms of the study system depend on too many biotic and abiotic factors to be described in a simulation model. However, we could estimate the benefits of a farmer using $P$. lagenophorae as biocontrol agent at various levels of severity of competition between $S$. vulgaris and $D$. carota. Moreover, we provided an outline for a general model describing the impact of the spatial dynamics of a natural enemy on plant competition.

\section{Acknowledgments}

We are grateful to Nilgün Sailer for her support of the experiment, and Kirsten Leiss, Jacco Wallinga, and Jacob Weiner for critical comments on a previous version of the manuscript. The study was supported by Grant NR. 3152380.97 of the Swiss National Science Foundation to JF. 


\section{References}

van den Bosch, F., Frinking, H.D., Metz, J.A.J., Zadoks, J.C., 1988. Focus expansion in plant disease. III: two experimental examples. Phytopathology 78, 919-925.

Firbank, L.G., Watkinson, A.R., 1990. On the effects of competition: from monocultures to mixtures. In: Grace, J.B., Tilman, D. (Eds.), Perspectives on Plant Competition. Academic Press, San Diego, pp. 165-192.

Frantzen, J., 2000. Disease epidemics and plant competition: control of Senecio vulgaris with Puccinia lagenophorae. Basic Appl. Ecol. 1, 141148.

Frantzen, J., van den Bosch, F., 2000. Spread of organisms: can travelling and dispersive waves be distinguished? Basic Appl. Ecol. 1, 83-92.

Frantzen, J., Müller-Schärer, H., 1999. Wintering of the biotrophic fungus Puccinia lagenophorae within the annual plant Senecio vulgaris: implications for biological weed control. Plant Pathol. 48, 483-490.

Frantzen, J., Paul, N.D., Müller-Schärer, H., 2001. The system management approach of biological weed control: some theoretical considerations and aspects of application. BioControl 46, 139-155.

Frantzen, J., Rossi, F., Müller-Schärer, H., 2002. Integration of biological control of common groundsel (Senecio vulgaris) and chemical control. Weed Sci. 50, 787-793.

Grace, B.S., Müller-Schärer, H., 2003. Biological control of Senecio vulgaris in carrots (Daucus carota) with the rust fungus Puccinia lagenophorae. Basic Appl. Ecol. 4, 375-384.
Law, R., Watkinson, A.R., 1987. Response-surface analysis of two species competition: an experiment on Phleum arenaria and Vulpia fasciculata. J. Ecol. 75, 871-886.

Leiss, K.A., Müller-Schärer, H., 2001. Population dynamics of the annual plant Senecio vulgaris in ruderal and agricultural habitats. Basic Appl. Ecol. 2, 53-64.

Müller-Schärer, H., Frantzen, J., 1996. An emerging system management approach for biological weed control in crops: Senecio vulgaris as a research model. Weed Res. 36, 483-491.

Paul, N.D., 1989. The effects of Puccinia lagenophorae on Senecio vulgaris in competition with Euphorbia peplus. J. Ecol. 77, 552-564.

Paul, N.D., Ayres, P.G., 1986. Seasonal effects on rust disease (Puccinia lagenophorae) of Senecio vulgaris. Symbiosis 2, 165-174.

Paul, N.D., Ayres, P.G., 1987. Effects of rust infection of Senecio vulgaris on competition with lettuce. Weed Res. 27, 431-441.

Paul, N.D., Ayres, P.G., 1990. Effects of interactions between nutrient supply and rust infection of Senecio vulgaris L. on competition with Capsella bursa-pastoris (L.) Medic. New Phytol. 114, 667-674.

Shigesada, N., Kawasaki, K., 1997. Biological Invasions: Theory and Practice. Oxford University Press, Oxford.

Watkinson, A.R., 1981. Interference in pure and mixed populations of Agrostemma githago. J. Appl. Ecol. 18, 967-976.

Watkinson, A.R., Freckleton, R.P., 1997. Quantifying the impact of arbuscular mycorrhiza on plant competition. J. Ecol. 85, 541-545.

Wyss, G.S. 1997. Quantitative Resistance in the Weed-Pathosystem Senecio vulgaris L.-Puccinia lagenophorae Cooke. Ph.D. thesis, ETH Zürich, Zürich. 